\title{
Australian Apparel Anthropometric 3D Database (AAA3D): A Collaborative Approach
}

\author{
Kate KENNEDY ${ }^{a}$, Jo KELLOCK ${ }^{b}$, Olga TROYNIKOV ${ }^{a}$ \\ ${ }^{a}$ RMIT University, Melbourne, Australia, ${ }^{b}$ Council of Textiles and Fashion Industries of Australia
}

\begin{abstract}
In recent years there has been a steady development of 3D body scanning research and equipment infrastructure within Australian Universities; i.e. RMIT University, the University of South Australia (UniSA), Curtain University in West Australia, and the Australian Institute of Sport (AIS). The newly established Textile and Fashion Hub; a collaboration between the Australian Government/AusIndustry, Council of Textiles and Fashion Industries of Australia Ltd (TFIA) and the Kangan Institute and the aims to provide a national network of textile and apparel entrepreneurs and manufacturers with access to $3 \mathrm{D}$ scanning technology.
\end{abstract}

Historically large scale 3D body scanning projects have been undertaken using a preferred technology model for data capture and divided between white light and laser formats. The opportunity exists with the current scanner infrastructure within Australian research institutions and industry partnerships to undertake a collaborative approach to anthropometric 3D data collection suitable for apparel design and development using a variety of scanning equipment. Protocols for capturing data according to anthropometric requirements, data sharing and database parameters need to be established from the outset. Such a model would allow for a series of small to medium scale scanning surveys to be undertaken concurrently and successively according to the specific needs of textile and apparel companies and consumers. Hypothetically, pooled data could create a shared resource to assist a range of anthropometric applications for textile and apparel innovation. The establishment of the Australian National Broadband Network (NBN) will provide more businesses, including those in regional areas, with the opportunity to participate.

This research is a preliminary trial to investigate a benchmarking and scan capture protocol between the white light TC ${ }^{2}$ NX16 scanner (at RMIT) and the laser Spacevision Cartesia 3D portable body scanner (at the Textile and Fashion Hub), in Melbourne, Australia.

Keywords: 3D body scanning, anthropometric data, cloud point data.

\section{Introduction}

This research was undertaken to establish a trial benchmarking and scan capture protocol between the white light TC $^{2}$ NX16 scanner at RMIT University and the laser Spacevision Cartesia 3D portable body scanner at the Textile and Fashion Hub. The investigation aimed to provide a proof of concept report to validate if cloud point data and extracted measurements from both scanners can be pooled to establish an Australian Apparel Anthropometric 3D Database.

\section{Other scanning projects}

The Australian apparel industry operates in a state of anthropometric anomie, with no reliable data or apparel size standards to inform the industry or consumers. There are no published standards for male and female apparel. Industry approaches to size definition are based on ad hoc methods derived from outdated size standards and inherited trade practice. Attempts to provide solutions to the lack of anthropometric data resources were recommended in 2008, The Australian Government's 2008 review of the Textile Clothing and Footwear (TCF) industry. The review document, Building Innovative Capability, highlighted the need for anthropometric data to assist the industry:

A new Australia National Sizing Standard for clothing and footwear is long overdue and should be developed as a matter of urgency, with a funding allocation of $\$ 5$ million, to meet the changing needs of customers and ensure that Australia TCF firms and organisations are not placed at a competitive disadvantage [1]. 
In spite of this recommendation and a later Government response to the Building Innovative Capability report, the May 2009 federal budget announced 'innovation and renewal in the Australian textiles, clothing and footwear (TCF) industries' by commissioning ...

the TCF Industries Innovation Council to provide further advice on the introduction of a voluntary ethical quality mark, voluntary national sizing standards for clothing and footwear, and a national anthropometric (human measurement) database; ... [2].

This recommendation has not eventuated. Thus this research seeks to investigate if scanning surveys can feasibly be undertaken using compatible 3D body scanners according to the specific needs of textile and apparel companies.

The Textile and Fashion Hub is a collaboration between the Australian Government/AusIndustry, Council of Textiles and Fashion Industries of Australia Ltd (TFIA) and the Kangan Institute to assist the needs of small to medium enterprises (SME's). In 2005/06, 24.5\% of TCF firms employed between 1 4 people [3]. The Hub facility has primary been established as 'a space for SMEs to conveniently access resources, knowledge, leading edge equipment, capital and experience [4]. The project specifically seeks to develop collaborative SME clusters. The TFIA brings an extensive membership network to the project as a national peak body organization for the TCF industry.

The Textile and Fashion Hub is linked by various TFIA membership collaborations via industry specific training in new technology applications. This includes collaborative research with Universities in Australia to foster industry innovation. RMIT University, as a member of the TFIA, has an affiliation to the Textile and Fashion Hub and has undertaken this research as an initial CAD cluster project.

Thus the concept of a collaborative approach to anthropometric 3D data collection using a variety of scanning equipment has the potential to create a shared database model for SME cluster participants as well as the broader industry. Scan surveys can therefore be conducted on an individual needs bases according to specific SME target markets. The establishment of the Australian National Broadband Network (NBN) will provide more businesses, including those in regional areas, with the opportunity to participate [5].

\section{Methodology}

This research is intended as first stage validation testing using two different types of scanner, i.e., the NX16 TC $^{2}$ white light scanner at RMIT University in Melbourne and the laser Spacevision Cartesia 3D portable body scanner at the Textile and Fashion Hub. The primary intension is to compare scan data and develop a scanning protocol that can be used by potential cluster participants in various locations in Australia. Thus the traceability of both scanner types' calibration protocol is a key requirement. Measurements from both scanners were extracted using the $\mathrm{TC}^{2}$ Measurement Extraction Software.

\subsection{Scan Tests}

The testing involved two test conditions. The first was using a fixed object mannequin and the second using a human subject. As the intension of this study is to develop a model that will allow for future participation from other interested parties with similar scanning technologies, the test mannequin was chosen from a commercially available range. The mannequin, a relatively inexpensive plastic form with a balanced stance, head and full limbs, is available as a stock item throughout Australia [6].

To prepare for the optimum underarm scanner view position an adjustment was made to the arm position on the mannequin. The arm angle was adjusted to conform to the arm angle position controlled by the NX16 scanner hand hold position. The NX16 scanner positions the arms at approximately 20 degrees (Fig 1 ) from the side body. Landmarks (adhesive dots) were also placed on the mannequin to define body levels. Positions were referenced according ISO 8559:1998 Garment construction and anthropometric survey - Body dimensions measurement protocols [7] with reference to (CAESAR), Final Report. Volume II: Descriptions [8], for supporting descriptions (Fig.2) Manual measurements were taken at these points on the mannequin according to ISO 8559. The NX16 scans were capture using an illuminate image function to visually show landmarks. The Cartesia scanner image capture is with an image quality that shows manual markings. 


\subsection{Limitations to study}

Further preparation of the mannequin was required after the initial testing with the Cartesia scanner showed a high level of data cloudpoint flare. As the mannequin is made from a translucent plastic it possibly causes a level of surface flare creating some data distortion from the scanner's laser. To address this error the mannequin was dressed in opaque hose on the lower body, and masking tape was applied to the chest area. The NX16 scanner did not show any cloudpoint flare errors.

In addition of the Cartesia scanner is located at the Textile and Fashion Hub in a temporary position; in a small room with an unstable environment for both light and temperature. The scan capture time for the Cartesia scanner is 2 seconds with the NX16 at 12 seconds.

\subsection{Measurement protocols}

Measurements were extracted from ten (10) locations on the body, according to ISO 8559 measurement definitions. With ten scans captured from each test set e.g.:

Test $1 \mathrm{a}=$ Mannequin $\times 10$ from Cartesia scanner

Test $1 \mathrm{~b}=$ Mannequin $\times 10$ from NX16

Test $2 \mathrm{a}=$ Live subject $\times 10$ from Cartesia scanner

Test $2 \mathrm{~b}=$ Live subject $\times 10$ from NX16

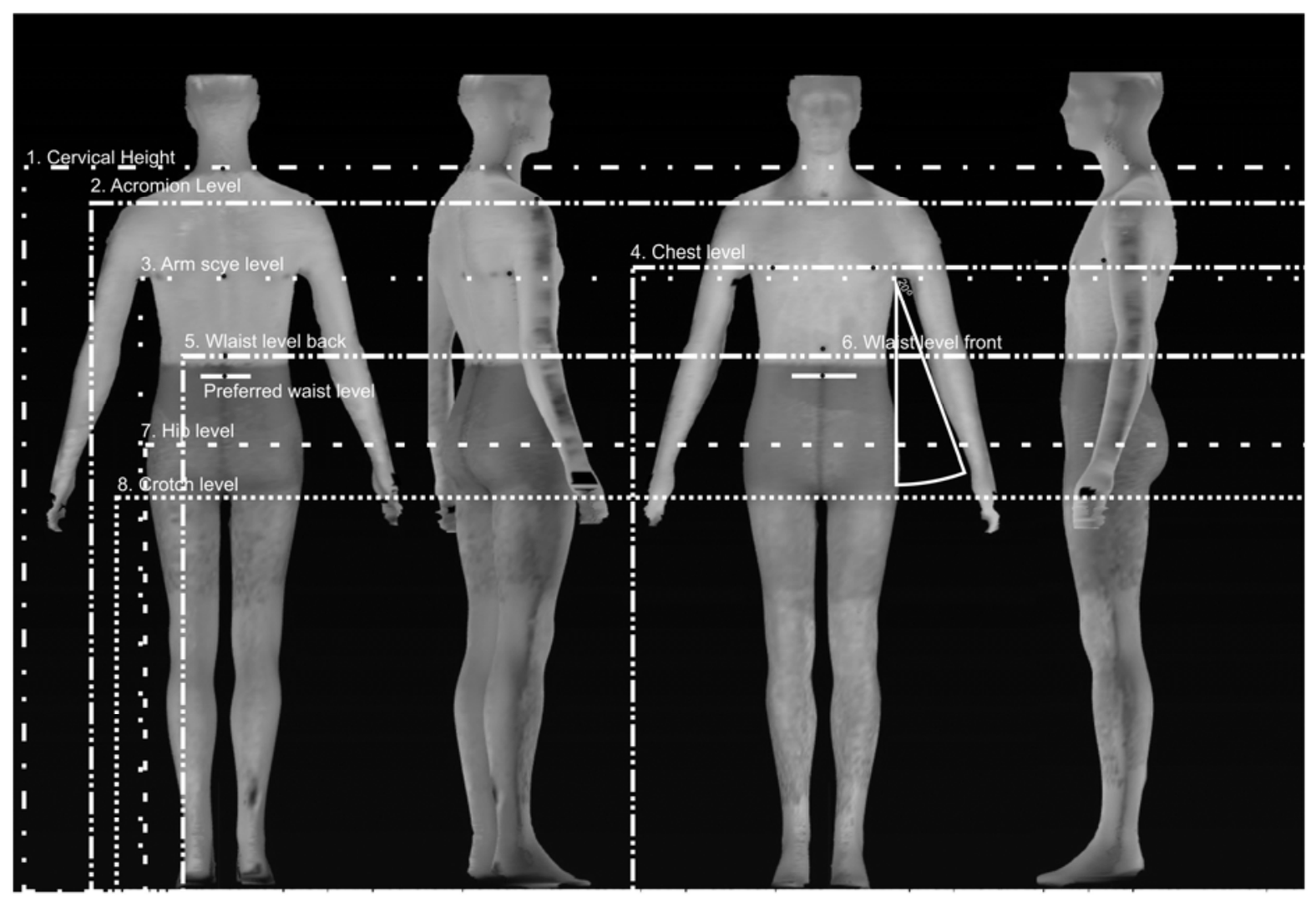

Fig 1: Body Level Detail for marker positions 


\begin{tabular}{|c|c|c|c|c|}
\hline $\begin{array}{l}\text { Level } \\
\text { Markers }\end{array}$ & $\begin{array}{l}\text { Measurement } \\
\text { Levels }\end{array}$ & $\begin{array}{l}\text { Level measured from floor ( } 0 \\
\text { point) vertical distance to: }\end{array}$ & Reference: & $\begin{array}{l}\text { Body } \\
\text { Plane }\end{array}$ \\
\hline 1 & $\begin{array}{l}\text { cervical height - } \\
\text { nape }\end{array}$ & seventh cervical vertebra & $\begin{array}{l}\text { ISO8559:2.2.12 (modified non } \\
\text { contoured) }\end{array}$ & posterior \\
\hline 2 & acromion level & $\begin{array}{l}\text { most prominent point of the } \\
\text { acromion at the shoulder -right }\end{array}$ & Caesar:ACROMION RIGHT p9 & lateral \\
\hline 3 & arm scye level & arm scye level - right & $\begin{array}{l}\text { armpit flesh fold right and left } \\
\text { Caesar: Axillia Fold }\end{array}$ & posterior \\
\hline 4 & bust/chest level & $\begin{array}{l}\text { for women - the most prominent } \\
\text { point of the bust. for men - the } \\
\text { centre of the nipple }\end{array}$ & $\begin{array}{l}\text { Caesar: Thelion/Bustpoint, Left } \\
\text { and Right p16 }\end{array}$ & anterior \\
\hline 5 & $\begin{array}{l}\text { waist level - } \\
\text { centre back }\end{array}$ & back waist position on small of back & ISO8559:2.1.11 & posterior \\
\hline 6 & $\begin{array}{l}\text { waist level- } \\
\text { centre front }\end{array}$ & $\begin{array}{l}\text { centre front waist point } \\
\text { (anterior) }\end{array}$ & ISO8559:2.1.11 & anterior \\
\hline 7 & hip level & $\begin{array}{l}\text { most prominent part of the trunk } \\
\text { (may be below the seat level) }\end{array}$ & $\begin{array}{l}\text { level defined } \\
\text { ISO8559:2.2.4 } \\
\text { Caesar } 24 \text { p54 }\end{array}$ & anterior \\
\hline 8 & inside leg & $\begin{array}{l}\text { crotch height - distance between } \\
\text { the crotch and the ground, feet } \\
\text { slightly apart. }\end{array}$ & ISO8559:2.2.27 anterior view & anterior \\
\hline
\end{tabular}

Fig 2: Body Level Detail for marker definitions

\begin{tabular}{|c|c|}
\hline $\begin{array}{l}\text { Body Dim } \\
\text { ISO } 8559 \text { / Other }\end{array}$ & Body Location \\
\hline 1. $\quad$ cervical height: ISO8559 2.2.12 & $\begin{array}{l}\text { Measured from the } 7 \text { th cervical }(7 \mathrm{C}) \text { vertebra, following the contour of } \\
\text { the rear spinal column to the hips and then vertical to the ground. }\end{array}$ \\
\hline 3. $\quad$ scye depth: ISO8559 2.2.9 & $\begin{array}{l}\text { Measured on the back, vertical distance from nape to scye level } \\
\text { (contour).(from level } 1 \text { to } 3 \text { ) }\end{array}$ \\
\hline $\begin{array}{ll}\text { 4. nape to waist centre back (CB): ISO8559 } \\
\text { 2.2.10 }\end{array}$ & $\begin{array}{l}\text { Measured on the back, vertical distance from nape (7C) to CB waist } \\
\text { level (contour). (from level } 1 \text { to } 5 \text { ) }\end{array}$ \\
\hline 5. nape to waist over bust: ISO8559: 2.2 .14 & $\begin{array}{l}\text { Distance from nape measured on the body tightly along the neck base } \\
\text { over the shoulder and the bust point to the CF waist level (from level } 1 \\
\text { to } 6 \text { ). }\end{array}$ \\
\hline 6. $\quad$ across back: ISO8559:2.1.6 & $\begin{array}{l}\text { Distance measured horizontally across the back from back flesh fold to } \\
\text { back flesh fold across shoulder blades }\end{array}$ \\
\hline 7. $\quad$ arm length: ISO8559: 2.2.22 (modified) & $\begin{array}{l}\text { Distance measured from acromion over the elbow point to carpus with } \\
\text { arm relaxed at the side. }\end{array}$ \\
\hline 8. $\quad$ total bust/chest: ISO85592.1.7/8 & $\begin{array}{l}\text { Circumference measured around the most prominent part of the chest } \\
\text { at the chest/bust level. }\end{array}$ \\
\hline 9. $\quad$ waist ISO8559: 2.1.11 & The girth of the natural waist line.. \\
\hline 10. hip -ISO8559:2.1.12 & Circumference measured at the most prominent part of the \\
\hline 11. inside leg: ISO8559: 2.2 .27 & $\begin{array}{l}\text { Distance measured at the inside of the leg from the crotch level to the } \\
\text { floor with legs apart. }\end{array}$ \\
\hline
\end{tabular}

Fig 3: Primary measurement points for scan measurement extraction

\begin{tabular}{|l|l|}
\hline $\begin{array}{l}\text { nape to bust/chest: } \\
\text { ISO8559 2.2.13 }\end{array}$ & $\begin{array}{l}\text { Distance from nape measured on the body tightly along the neck base } \\
\text { over the shoulder to the bust point (from level 1 to 4). }\end{array}$ \\
\hline scye width: & $\begin{array}{l}\text { Distance measured under the arm from the back flesh fold to the front } \\
\text { flesh fold. }\end{array}$ \\
\hline $\begin{array}{l}\text { biacromial width: } \\
\text { ISO8559: } 2.1 .5\end{array}$ & $\begin{array}{l}\text { Distance measured horizontally across the back from acromion to } \\
\text { acromion. }\end{array}$ \\
\hline waist - preferred & $\begin{array}{l}\text { Circumference measured at the 'preferred' waist level. } \\
\text { Note: Preferred waist level is established by the subject, who places an } \\
\text { elastic band at the level he or she would prefer to wear the waist of their } \\
\text { pants. }\end{array}$ \\
\hline $\begin{array}{l}\text { waist to hip depth } \\
\text { ISO8559:2.2.17 }\end{array}$ & $\begin{array}{l}\text { Distance measured at the side of the body from waist level over the hip } \\
\text { curve to the level of most prominent point below the waist (hip level). }\end{array}$ \\
\hline $\begin{array}{l}\text { waist to floor- outside leg length: } \\
\text { ISO8559:2.2.25 }\end{array}$ & Distance measured at the side of the body from waist level to floor level. \\
\hline
\end{tabular}

Fig 4: Secondary measurement points for reference 


\section{Analysis}

As a benchmark test: the primary assessment of the test mannequin's extracted scanner measurements against the manual measurements shows the range of measurement variation and the degree of variance within the ten scans. (Fig 5). Test 1a compares the Cartesia scanner results to the manual measurements. Test $1 \mathrm{~b}$ compares the NX16 scanner extracted measurement results to the manual measurements. The degree of acceptance of the variation (from scan to manual measurement) was assessed according to a tolerance factor of plus or minus (+/-) $1.5 \%$ of the mean of each of the ten measurement locations. Test 1a (Cartesia) shows that seven out of the ten $(70 \%)$ measurements with a result that is outside the acceptable tolerance and Test $1 \mathrm{~b}$ (NX16) show a five out of ten $(50 \%)$.

The biggest measurement variation is shown to be at the scye depth (ISO8559: 2.2.9) measurement point. Both scanner type extracted measurements show a variation outside the $1.5 \%(+/-)$ tolerance. Test 1a (Cartesia) shows the difference from the mean measurement for scye depth, from the manual measurement tolerance to be $-22.2 \mathrm{~mm}$ outside the acceptable tolerance. Test $1 \mathrm{~b}$ (NX16) to be -31.9 $\mathrm{mm}$ (fig 4). This measurement point is the most extreme variation to the manual measurement of all ten measurement points. The arm length (ISO8559: 2.2.22), across back (ISO8559: 2.1.6), nape to waist over bust (ISO8559: 2.2.14) show as a common error in both scanner measurements.

The position of the scye measurement point has a direct relationship to the other upper body measurement, the across back (ISO8559: 2.1.6). This measurement has the greatest variation from the manual and shows the highest degree of variance (CV) within the ten scans (NX16). The inside leg measurement (ISO8559:2.2.27) extracted from the Cartesia scanner is a consistently larger measurement than the manual and NX16 measurements and requires further investigation.

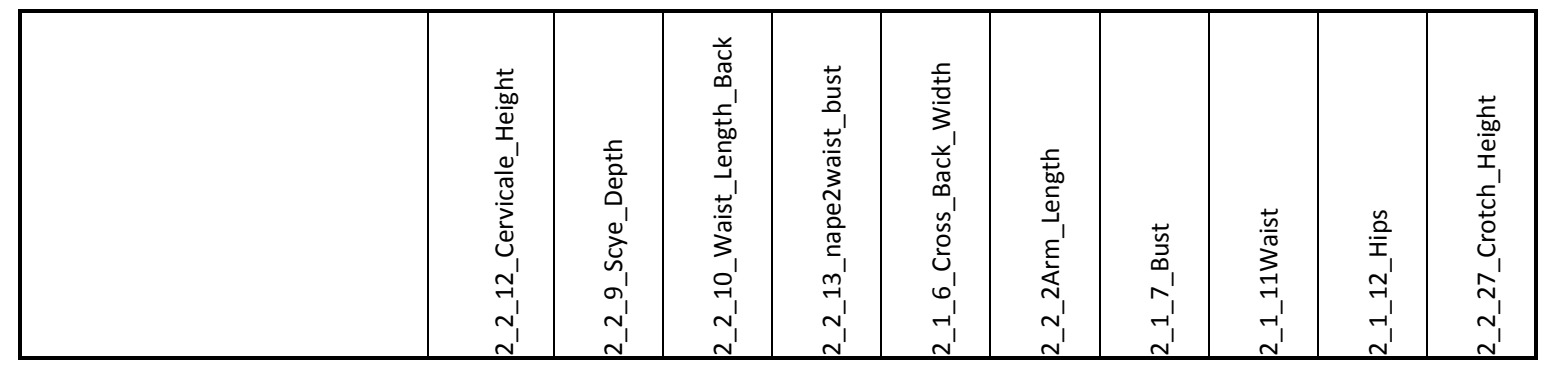

Test 1a (Cartesia) Mannequin $\times 10$ scans

\begin{tabular}{|r|r|r|r|r|r|r|r|r|r|r|}
\hline Mean & $\mathbf{1 6 7 8 . 1}$ & $\mathbf{2 0 8 . 6}$ & $\mathbf{4 4 5 . 3}$ & $\mathbf{5 4 0 . 6}$ & $\mathbf{3 7 8 . 9}$ & $\mathbf{6 8 7 . 8}$ & $\mathbf{9 6 6 . 5}$ & $\mathbf{7 4 9 . 2}$ & $\mathbf{9 6 7 . 4}$ & $\mathbf{9 0 1 . 7}$ \\
\hline STDev & 9.7 & 5.3 & 8.5 & 9.3 & 5.1 & 3.3 & 4.1 & 2.7 & 0.8 & 2.9 \\
\hline $\mathrm{CV}$ & 0.6 & 2.6 & 1.9 & 1.7 & 1.4 & 0.5 & 0.4 & 0.4 & 0.1 & 0.3 \\
\hline max/min dif & 29.4 & 16.5 & 29.3 & 32.2 & 16.5 & 9.6 & 13.6 & 9.2 & 2.2 & 10.2 \\
\hline Manual Measurements & $\mathbf{1 6 4 0 . 0}$ & $\mathbf{2 5 5 . 0}$ & $\mathbf{4 4 0 . 0}$ & $\mathbf{5 3 0 . 0}$ & $\mathbf{3 6 5 . 0}$ & $\mathbf{6 4 0 . 0}$ & $\mathbf{9 7 0 . 0}$ & $\mathbf{7 7 0 . 0}$ & $\mathbf{9 8 0 . 0}$ & $\mathbf{8 8 0 . 0}$ \\
\hline \hline dif from manual & 38.1 & $\mathbf{- 4 6 . 4}$ & 5.3 & 10.6 & 13.9 & 47.8 & -3.5 & -20.8 & -12.6 & 21.7 \\
\hline tol in mms +/- 1.5 \% of mean & 25.2 & 3.1 & 6.7 & 8.1 & 5.7 & 10.3 & 14.5 & 11.2 & 14.5 & 13.5 \\
\hline \% tolerance & 1.5 & 1.5 & 1.5 & 1.5 & 1.5 & 1.5 & 1.5 & 1.5 & 1.5 & 1.5 \\
\hline \% variation actual & $\mathbf{2 . 3}$ & $\mathbf{- 2 2 . 2}$ & $\mathbf{1 . 2}$ & $\mathbf{2 . 0}$ & $\mathbf{3 . 7}$ & $\mathbf{6 . 9}$ & $\mathbf{- 0 . 4}$ & $\mathbf{- 2 . 8}$ & $\mathbf{- 1 . 3}$ & $\mathbf{2 . 4}$ \\
\hline$>$ tolerance & $*$ & $*$ & & $*$ & $*$ & $*$ & & $*$ & & $*$ \\
\hline
\end{tabular}




\begin{tabular}{|r|r|r|r|r|r|r|r|r|r|r|}
\hline \multicolumn{1}{|c|}{ Test 1b (NX16) Mannequin x 10 scans } \\
\hline Mean & $\mathbf{1 6 6 4 . 8}$ & $\mathbf{1 9 3 . 3}$ & $\mathbf{4 4 7 . 0}$ & $\mathbf{5 4 1 . 4}$ & $\mathbf{3 9 5 . 8}$ & $\mathbf{6 7 2 . 5}$ & $\mathbf{9 7 1 . 0}$ & $\mathbf{7 6 1 . 2}$ & $\mathbf{9 7 5 . 8}$ & $\mathbf{8 8 5 . 2}$ \\
\hline STDev & 1.7 & 4.9 & 3.1 & 2.8 & 24.8 & 7.2 & 11.9 & 0.5 & 0.5 & 1.9 \\
\hline max/min dif & 5.7 & 12.8 & 9.6 & 8.0 & 90.6 & 19.3 & 31.1 & 1.6 & 1.4 & 5.9 \\
\hline CV & 0.1 & 2.5 & 0.7 & 0.5 & 6.3 & 1.1 & 1.2 & 0.1 & 0.0 & 0.2 \\
\hline Manual Measurements & $\mathbf{1 6 4 0 . 0}$ & $\mathbf{2 5 5 . 0}$ & $\mathbf{4 4 0 . 0}$ & $\mathbf{5 3 0 . 0}$ & $\mathbf{3 6 5 . 0}$ & $\mathbf{6 4 0 . 0}$ & $\mathbf{9 7 0 . 0}$ & $\mathbf{7 7 0 . 0}$ & $\mathbf{9 8 0 . 0}$ & $\mathbf{8 8 0 . 0}$ \\
\hline \hline dif from manual & 24.8 & -61.7 & 7.0 & 11.4 & 30.8 & 32.5 & 1.0 & -8.8 & -4.2 & 5.2 \\
\hline tol in mms +/- 1.5 \% of mean & 25.0 & 2.9 & 6.7 & 8.1 & 5.9 & 10.1 & 14.6 & 11.4 & 14.6 & 13.3 \\
\hline \% tolerance & 1.5 & 1.5 & 1.5 & 1.5 & 1.5 & 1.5 & 1.5 & 1.5 & 1.5 & 1.5 \\
\hline$>$ variation actual & $\mathbf{1 . 5}$ & $\mathbf{- 3 1 . 9}$ & $\mathbf{1 . 6}$ & $\mathbf{2 . 1}$ & $\mathbf{7 . 8}$ & $\mathbf{4 . 8}$ & $\mathbf{0 . 1}$ & $\mathbf{- 1 . 2}$ & $\mathbf{- 0 . 4}$ & $\mathbf{0 . 6}$ \\
\hline$>$ tolerance & & $*$ & $*$ & $*$ & $*$ & $*$ & & & & \\
\hline
\end{tabular}

Fig 5: Summary of extracted scanner measurements Cartesia/NX16 to manual measurement

$50 \%$ of the NX16 scanners extracted measurements are within tolerance, while only $30 \%$ of the Cartesia scanners are within the acceptable range of the manual measurement. When considering this result it is important to consider the error in locating the correct landmark points for the $7^{\text {th }}$ Cervical and the Acromion on a mannequin that does not have anthropometric locators. Thus the locations of the landmark reference points on the mannequin may not necessarily comply directly with the $\mathrm{TC}^{2}$ measurement extraction software point definitions. A visual audit of the scans shows areas of data point misalignment to the manual landmarks. The scan data points can be realigned to landmark data points to reassess measurement accuracy between both scanners.

From the results shown (Fig 5), it is necessary to edit the 3D data measurement points that are outside the acceptable tolerance of $+/-1.5 \%$. For the Cartesia scans (test 1a) these seven points are: cervical height $(2.3 \mathrm{~mm})$, scye depth $(-22.2 \mathrm{~mm})$, nape to waist_bust $(2.0 \mathrm{~mm})$, across back width $(3.7 \mathrm{~mm}) \mathrm{arm}$ length $(6.9 \mathrm{~mm})$, waist $(-2.8 \mathrm{~mm})$ and the crotch_height $(2.4 \mathrm{~mm})$. For the NX16 scans these five points are: scye depth $(-31.9 \mathrm{~mm})$, center back length $(1.6 \mathrm{~mm})$, nape to waist_bust $(2.1 \mathrm{~mm})$, across back width $(7.8 \mathrm{~mm})$ and the arm length $(4.8 \mathrm{~mm})$. 


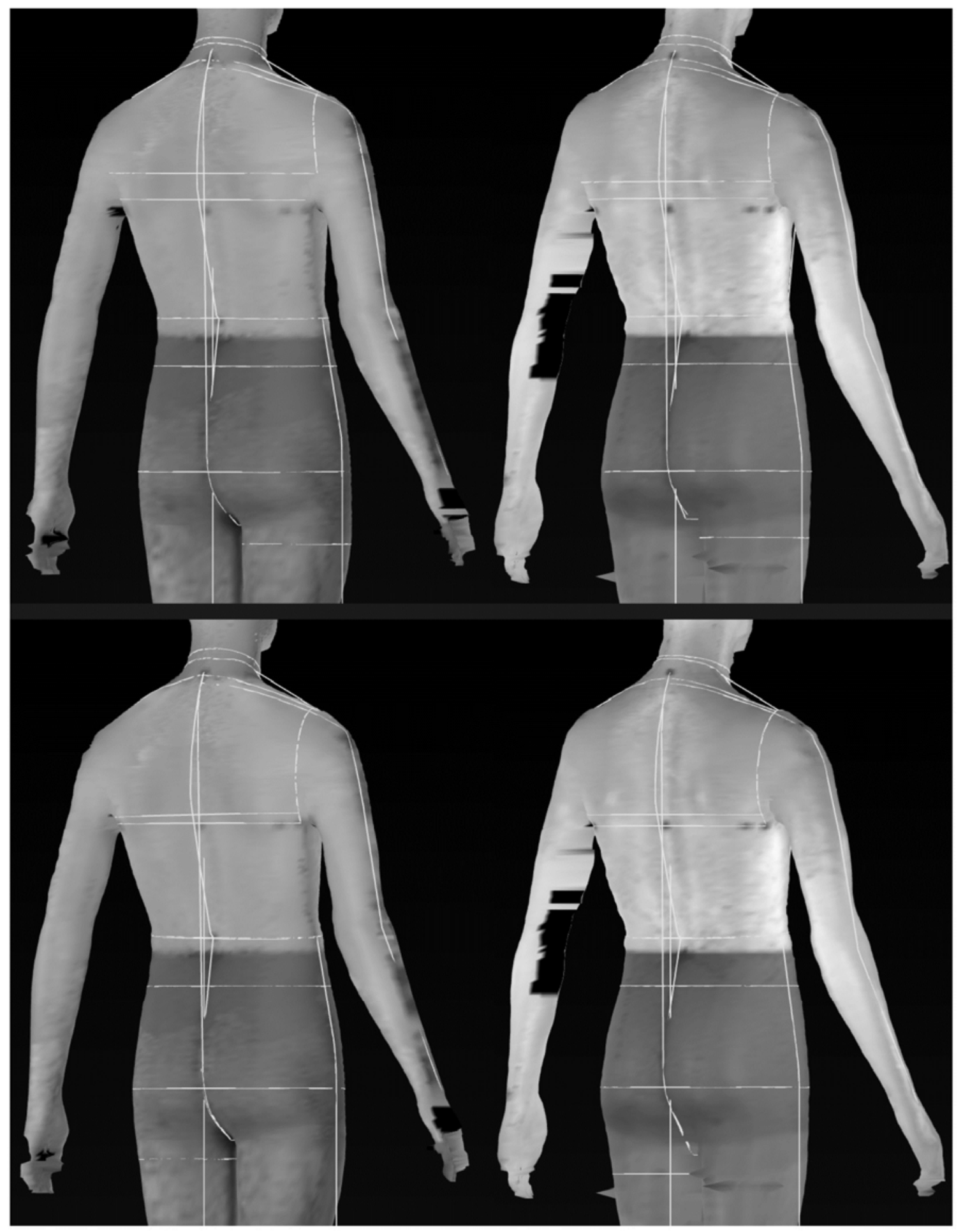

Fig 6: NX16(top left) Cartesia (top right) measurement and landmark points as scanned NX16(bottom left) Cartesia (bottom right) with points modified to match landmarks 


\begin{tabular}{|c|c|c|c|c|c|c|c|c|c|c|}
\hline & $\begin{array}{l}\overbrace{1} \\
\sim_{1}^{\prime} \\
\sim_{1}^{\prime}\end{array}$ & $\begin{array}{l}\sigma_{1} \\
\tilde{N}_{1} \\
\tilde{N}^{\prime}\end{array}$ & $\begin{array}{l}\text { 우 } \\
{ }_{1} \\
\sim_{1} \\
\sim^{\prime} \\
\end{array}$ & $\begin{array}{l}m_{1} \\
\sim_{1}^{\prime} \\
\sim_{1}^{\prime} \\
N^{\prime}\end{array}$ & $\begin{array}{l}\sigma_{1} \\
r_{1} \\
\sim^{\prime} \\
\end{array}$ & $\begin{array}{l}N_{1} \\
\sim_{1} \\
\sim^{\prime} \\
\end{array}$ & $\begin{array}{l}\hat{n}_{1}^{\prime} \\
\vec{\sim}_{1}^{\prime} \\
\end{array}$ & $\begin{array}{l}z_{1} \\
\vec{r}_{1} \\
\sim_{1}\end{array}$ & $\begin{array}{l}\sim_{1} \\
\sim_{1}^{\prime} \\
\sim \\
\sim\end{array}$ & $\begin{array}{l}\hat{N}_{1} \\
\sim_{1}^{\prime} \\
\sim^{\prime}\end{array}$ \\
\hline Manual Measurements & 1640. & 255.0 & 440.0 & 530.0 & 365.0 & 640.0 & 970.0 & 770.0 & 980.0 & 880.0 \\
\hline Test 1a mod (Cartesia) Mean & 1662.0 & 242.4 & 442.4 & 539.8 & 364.9 & 655.0 & 969.7 & 749.0 & 967.4 & 885.7 \\
\hline STDev & 1.8 & 1.4 & 2.1 & 7.5 & 2.8 & 8.1 & 5.1 & 2.0 & 0.9 & 8.0 \\
\hline $\max / \min$ dif & 5.1 & 3.8 & 5.6 & 17.6 & 7.8 & 20.3 & 13.5 & 5.4 & 2.2 & 21.2 \\
\hline coefficient of variation & 0.1 & 0.6 & 0.5 & 1.4 & 0.8 & 1.2 & 0.5 & 0.3 & 0.1 & 0.9 \\
\hline dif from manual & 22.0 & -12.6 & 2.4 & 9.8 & -0.1 & 15 & -0.3 & -21.0 & -12.6 & 5.7 \\
\hline tol in $\mathrm{mms}+/-1.5 \%$ of mean & 24.9 & 3.6 & 6.6 & 8.1 & 5.5 & 9.8 & 14.5 & 11.2 & 14.5 & 13.3 \\
\hline$\%$ tolerance & 1.5 & 1.5 & 1.5 & 1.5 & 1.5 & 1.5 & 1.5 & 1.5 & 1.5 & 1.5 \\
\hline$\%$ variation actual & 1.3 & -5.2 & 0.5 & 1.8 & 0.0 & 2.3 & 0.0 & -2.8 & -1.3 & 0.6 \\
\hline
\end{tabular}

\begin{tabular}{|r|r|r|r|r|r|r|r|r|r|r|}
\hline Test 1b mod (NX16) Mean & 1646.9 & 236.8 & 437.5 & 542.3 & 371.0 & 636.3 & 979.9 & 760.7 & 975.9 & 884.9 \\
\hline STDev & 1.7 & 1.5 & 4.0 & 4.4 & 4.4 & 8.5 & 6.0 & 1.4 & 0.4 & 2.0 \\
\hline max/min dif & 4.3 & 4.1 & 12.9 & 11.7 & 12.2 & 24.6 & 15.9 & 4.2 & 1.3 & 5.9 \\
\hline coefficient of variation & 0.1 & 0.6 & 0.9 & 0.8 & 1.2 & 1.3 & 0.6 & 0.2 & 0.0 & 0.2 \\
\hline dif from manual & 6.9 & -18.2 & -2.5 & 12.3 & 6.0 & -3.7 & 9.9 & -9.3 & -4.1 & 4.9 \\
\hline tol in mms +/- \% of mean & 24.7 & 3.6 & 6.6 & 8.1 & 5.6 & 9.5 & 14.7 & 11.4 & 14.6 & 13.3 \\
\hline \% tolerance & 1.5 & 1.5 & 1.5 & 1.5 & 1.5 & 1.5 & 1.5 & 1.5 & 1.5 & 1.5 \\
\hline \% variation actual & $\mathbf{0 . 4}$ & $\mathbf{- 7 . 7}$ & $\mathbf{- 0 . 6}$ & $\mathbf{2 . 3}$ & $\mathbf{1 . 6}$ & $\mathbf{- 0 . 6}$ & $\mathbf{1 . 0}$ & $\mathbf{- 1 . 2}$ & $\mathbf{- 0 . 4}$ & $\mathbf{0 . 6}$ \\
\hline
\end{tabular}

Fig 7: Summary of Cartesia / NX16 landmark adjusted scan measurement extractions to manual measurement

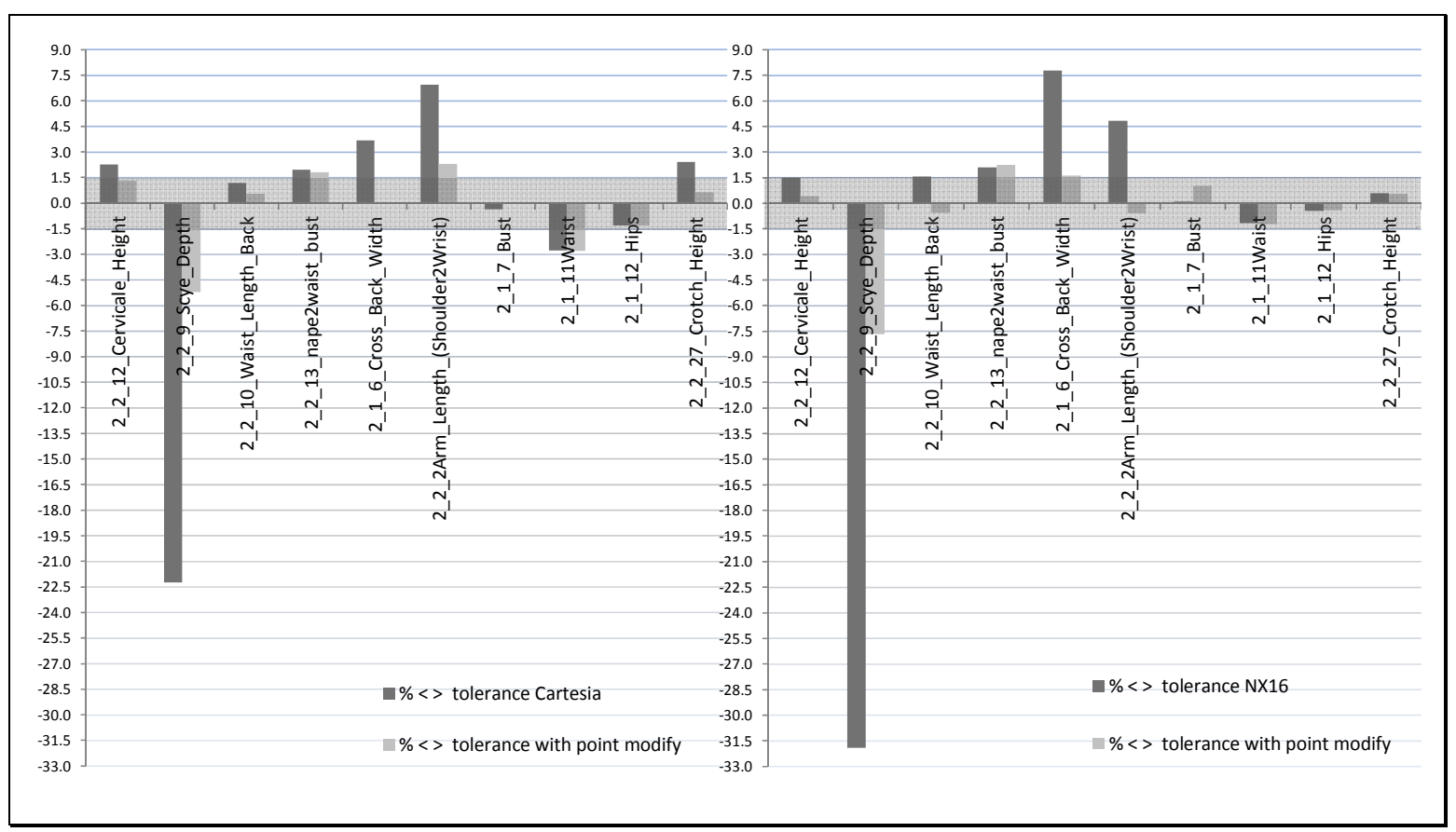

Fig 8: Cartesia / NX16\% variation actual_original scan measurement (Fig 5) against point modified (Fig 7) 
The comparison of the original scan measurements (Fig 8) to the modified points scan for both the Cartesia and NX16 scanner shows improved results. The Cartesia scans, when points were modified, improved from $70 \%$ outside acceptable tolerance to $40 \%$. Of the $40 \%$ the range of variation is greatly reduced; e.g. scye depth (2_2_9), from $-20.7 \%$ to $-3.7 \%$, arm length (2_2_2), from $5.4 \%$ to $0.8 \%$. The NX16 scans improved from $50 \%$ within tolerance to $80 \%$, with scye depth showing the greatest range from $-30.4 \%$ to $-6.2 \%$.

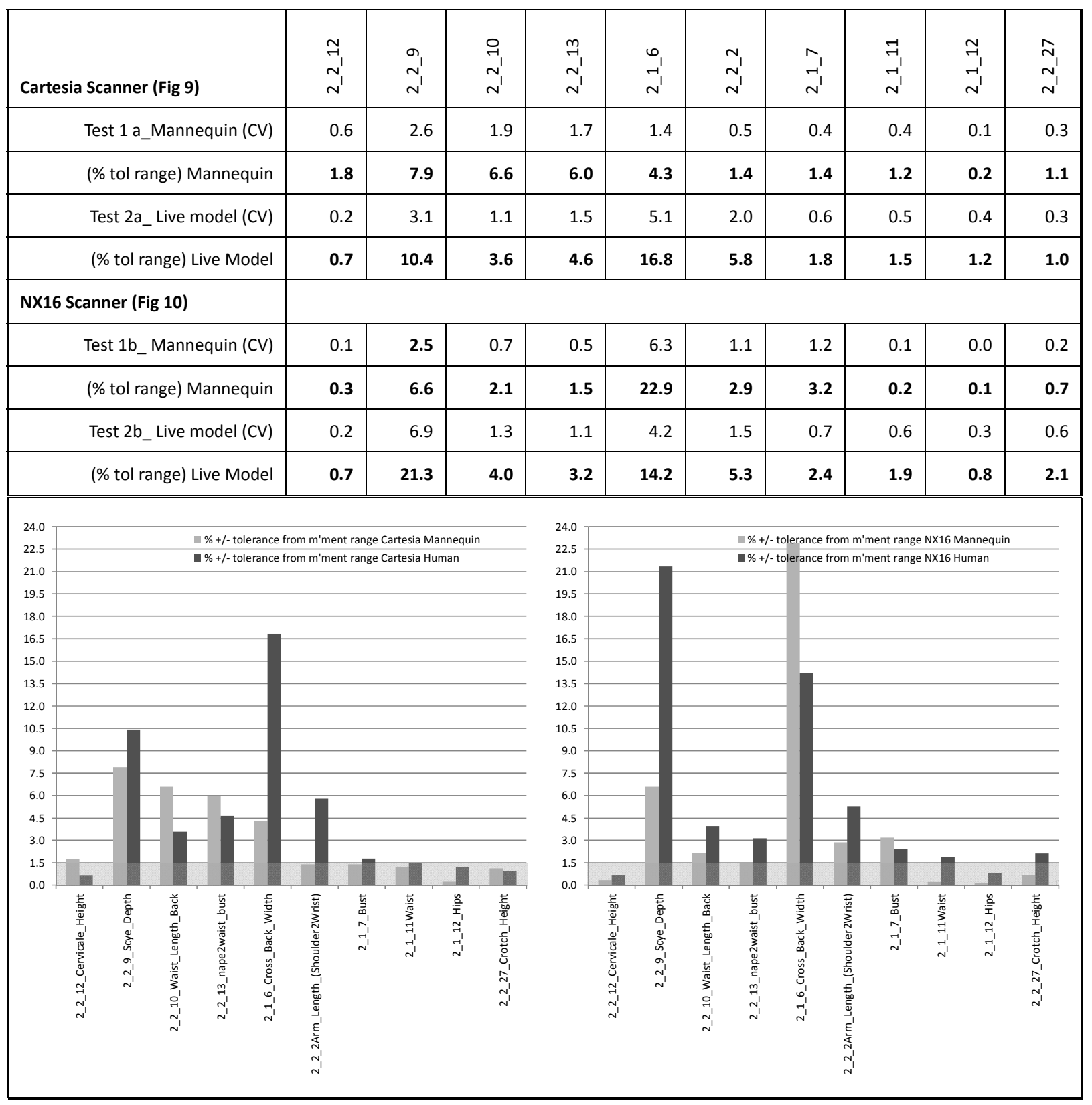

Fig 9: Comparison > \%1.5 tolerance error Cartesia scans mannequin (test 1a) to live model (test 2a)
Fig 10: Comparison > \%1.5 tolerance error NX16 scans mannequin (test 1 b) to live model (test

When comparing all primary scan tests (Fig 9_10), Cartesia scanner mannequin tests and live model test, and likewise for the NX16, the measurement areas that show a tolerance outside the benchmark $(1.5 \%)$ are consistent with previous results. Scye depth (2_2_9), across back (2_1_6), arm length (2_2_2), and nape to waist over bust (2_2_13) show significant tolerance errors. The bust (2_1_7), waist (2_1_11) and hip (2_1_12) the crotch height (2_2_27) are more consistent measurement points. 
When reviewing these results (fig 9_10), comparing them to the results with modified points (Fig 8), shows how modifying points can achieve a better outcome. The scans from the live model tests from both scanners would therefore require point modification to achieve the best result.

The comparisons of the girth measurements (Fig 9_Fig10) for the bust (2_1_7), waist (2_1_11), and hip (2_1_12) show a more consistent range for the Cartesia scan results. The difference in the scan capture time, 2 seconds (Cartesia) vs 12 seconds (NX16) may have an impact during scan capture for horizontal. The live model test from the NX16 shows a greater range than that shown on the mannequin.

\section{Conclusion}

As a preliminary test into the data compatibility of cloud point data and extracted measurements from two different scanners, white light $\mathrm{TC}^{2} \mathrm{NX} 16$ scanner and the laser Spacevision Cartesia 3D portable body scanner the results show a promising outcome. The primary scans captured on a mannequin and benchmarked against manual measurements show the range, compatibility and limitations of the tests. There are a number of factors to be considered.

The first consideration is for the capacity for a traceable scanner calibration protocol so that measurements can be validated. Both the $\mathrm{TC}^{2} \mathrm{NX} 16$ and Spacevision Cartesia scanner have a simple and recordable calibration protocol.

The second consideration is that the results show that a compatible data range can only be achieved from the use of physical landmarkers. This allows for the ability to visually inspect the scan images and match the 3D data points to the landmark points. Therefore the 3D image capture must have the capacity for image illumination during capture time. The scye depth area showed on both scanners to be a very necessary landmark point.

The tolerance percentage of plus or minus $1.5 \%(+/-)$, applied as the benchmark may perhaps be reviewed according to specific measurement locations. For example this percentage may not be a realistic amount to apply to body measurements that need a higher degree of flexibility such as the waist or chest. Further investigation into more specific calculations in the measurement extraction profiles in the $\mathrm{TC}^{2}$ software could also achieve a greater degree of accuracy. It is envisaged that the SME clusters with the fashion and Textile and Fashion Hub can further participate in developing best practice measurement protocols specific to the needs of the Australian TCF industry.

The final recommendation is that further investigation should be undertaken in the areas that provided some limitations to this study. The major area of further investigation being: how to stabilize the light and temperature environment for the Spacevision Cartesia scanner. As a portable scanner, this equipment is perfectly suited to easy relocation for a variety of scan capture scenarios e.g., small to medium enterprise scan surveys. Thus the development of a portable screen booth that provides a more stable environment would be recommended.

In taking these factors into account these preliminary tests show a positive result. Thus it would appear that it is feasible to allow for small to medium scale scanning surveys to be undertaken concurrently and successively. Hypothetically, pooled data could create a shared resource to assist a range of anthropometric applications for apparel innovation. The establishment of the Australian National Broadband Network (NBN) will provide more businesses, including those in regional areas, with the opportunity to participate in a collaborative scanning project to establish an Australian Apparel Anthropometric 3D Database. 


\section{References}

1. Green, R., (2008): "Building Innovative Capability: Review of the Australian Textile, Clothing and Footwear Industries". Dept: Innovation, Industry, Science and Research, Canberra: Commonwealth of Australia, p.111.

2. Carr, K. (Innovation Minister), (2009): "BUILDING INNOVATION IN TEXTILES, CLOTHING AND FOOTWEAR", media release, Parliament House, 12 May. Viewed 11 November 2009, http://minister.innovation.gov.au/Carr/Pages/BUILDINGINNOVATIONINTEXTILESCLOTHINGAN DFOOTWEAR.aspx.

3. Green, R., (2008): "Building Innovative Capability: Review of the Australian Textile, Clothing and Footwear Industries". Dept: Innovation, Industry, Science and Research, Canberra: Commonwealth of Australia, p 25.

4. http://www.tfia.com.au/hub . viewed 20 August 2012.

5. http://www.nbn.gov.au/ . viewed 20 August 2012.

6. http://www.meipicchi.com/index.php?mact=ProductManager,cntnt01,frontend_essentials,0\&cntnt0 1product_group_id=7\&cntnt01subgroup_id=23\&cntnt01 product_id $=160 \&$ cntnt 01 returnid $=20$ viewed 20 August 2012.

7. International Organization for Standardization, (1998): ISO 8559:1998 "Garment construction and anthropometric survey - Body dimensions". Switzerland.

8. Blackwell, S., Robinette, K. M., Boehmer, M., Fleming, S., Kelly, S., Brill, T., Hoeferlin, D., Burnsides, D. \& Dannen, H., (2002): "Civilian American and European Surface Anthropometry Resource (CAESAR), Final Report. VOLUME II: DESCRIPTIONS". DAYTON OHIO, pp.8-38. 